Monográfico: ENDOUROLOGÍA Y LÁSER

Arch. Esp. Urol., 61, 9 (1.023-1.027), 2008

\title{
PRÓSTATA Y LÁSER KTP
}

Carlos Hernández Fernández, David Subirá Rios, Gonzalo Bueno Chomón y Juan Tabares Jiménez.

Servicio de Urología. Hospital General Universitario Gregorio Marañón. Madrid. España.

Resumen.- OBJETIVO: Analizar en base a los datos de la literatura médica la seguridad y eficacia de la fotovaporización con Láser verde KTP en el tratamiento de la obstrucción urinaria causada por la hipertrofia benigna de próstata.

RESULTADOS: La mayor parte de los artículos consultados destacan la baja tasa de complicaciones, la eficacia y corta curva de aprendizaje de este tipo de vaporización. Teniendo como limitaciones que la mayor parte de dichos trabajos no son comparativos, el número de pacientes incluidos no es importante y sobre todo el seguimiento de muchos de ellos es corto.
CONCLUSIONES: La vaporización prostática con Láser KTP es hoy una alternativa a la RTU y más en pacientes con comorbilidad, por la baja tasa de complicaciones. Aunque asumiendo que es una tecnología cara y que precisa de un mayor seguimiento para confirmar el mantenimiento de los resultados obtenidos.

Palabras clave: HBP. Láser KPT. Láser verde. Láser.

Summary:- OBJECTIVES: To analyze safety and efficacy of KTP green laser photovaporization for the treatment of lower urinary tract obstruction caused by benign prostatic hyperplasia based on data from the medical literature.

RESULTS: Most articles consulted emphasize its low complication rate, efficacy, and short learning curve. They have the limitation that most papers are not comparative, the number of patients included is low, and follow-up in many of them short.

CONCLUSIONS: Prostatic vaporization with KTP laser is today an alternative to TUR, more in patients with comorbidities, due to its low complication rate. Nevertheless, we accept it is an expensive technology and more follow-up is required to confirm the results remain.

Keywords: BPH. KPT laser. Green laser. Laser.

\section{INTRODUCCIÓN}

La tecnología láser ha sido empleada, en la década de los 90, para mejorar la obstrucción urinaria secundaria al crecimiento prostático. Pero muchos de estos generadores de energía tuvieron que ser abandonados, poco tiempo después, por la importante sintomatología irritativa postquirúrgica que condicionaban, así como por la alta 
tasa de reintervenciones que precisaban los pacientes al no mejorar, en muchos de ellos la obstrucción urinaria que padecían. Todo ello generaba un aumento significativo del coste del procedimiento $(1,2)$.

En la década de los 90 se desarrolla el láser KTP que se basa en el ya conocido Láser de neodimio:YAG cuya luz se hace pasar por un cristal de potasio-titanylfosfato lo que le da una longitud de onda de $532 \mathrm{~nm}$ y le coloca en el segmento verde del espectro visual, de ahí que tome también el nombre de "láser verde".

Como características propias es de destacar que esta longitud de onda se absorbe, de forma selectiva, por aquellos tejidos que estando bien vascularizados son ricos en oxi-hemoglobina, mientras que su absorción por el agua es muy pequeña.

En consecuencia se genera una temperatura suficientemente alta como para vaporizar el tejido, pero con una penetración muy pequeña (1-2 mms) lo que permite una mínima dispersión de calor desde el punto de contacto (3). La irrigación permanente lava las burbujas producidas.

Se diseñaron estudios con perros con el fin de valorar su seguridad, utilizando primero potencias de $38 \mathrm{~W}$ pasando después a $60 \mathrm{~W}$. Dichos trabajos constataron que con ambos niveles de energía se conseguían incisiones a nivel del cuello vesical pero cuando la potencia se aumentaba disminuía el tiempo quirúrgico $(4,5)$.

Posteriormente es el grupo de la la Clínica Mayo el que en 1998 publica un artículo con la inclusión de 10 pacientes, tratados con Láser KTP, sin complicaciones significativas y con retirada de la sonda vesical a las 24 de la intervención (6).

Es a partir de entonces cuando esta tecnología se va expandiendo a distintos hospitales y paises, con la ventaja de que el instrumental que necesita para su utilización es similar al utilizado en cualquier Servicio de Urología, un cistoscopio 22-23 F con irrigación continua y un canal de trabajo que permita la introducción de la fibra láser.

Así mismo los equipos han ido aumentando su potencia y en la actualidad disponemos de generadores de alta potencia que alcanzan los $120 \mathrm{~W}$

\section{TÉCNICA QUIRÚRGICA}

La técnica anestésica puede ser indistintamente raquídea, general, o incluso combinando sedación con infiltración local prostática (7).

A través del cistoscopio previamente mencionado se introduce la fibra que, a través de un sistema de espejo, emite el haz de láser con un ángulo de $90^{\circ}$.

Después de una inspección visual para localizar los orificios ureterales, veru montanum e identificar la morfología prostática, se inicia el tratamiento intentando colo- car la punta del láser a una distancia del tejido no mayor de $1 \mathrm{~mm}$ de forma que la vaporización sea la idónea y disminuya el riesgo de carbonización empeorando la eficacia.

Como irrigación puede emplearse tanto suero salino como agua bidestilada, con lo que mantenemos una correcta visión además de lavar y exteriorizar las burbujas que se van formando. La aparición de estas burbujas es signo de una correcta vaporización del tejido prostático.

La mayoría de los urólogos prefieren iniciar el tratamiento a nivel del cuello vesical, retirándose paulatinamente hasta alcanzar el veru-montanum. Una vez creado un canal, que debe poner en evidencia las fibras trasversales del cuello vesical, se inicia la vaporización de los lóbulos laterales.

Es también interesante recalcar que el movimiento de la fibra no ha de ser como el asa de relector de proximal a distal, sino más bien un movimiento de rotación con un arco de $30-60^{\circ}$. Con la precaución de no acercar en exceso la energía a la óptica, ya que el calor podría fácilmente estropearla.

El procedimiento es prácticamente exangüe, pero aún así, en ocasiones han de coagularse pequeños vasos sangrantes, para ello basta con alejar la fibra unos milímetros de la zona a coagular, o bien bajar la potencia del equipo a cifras de 30-60 W.

Una vez acabada la vaporización y confirmada una correcta hemostasia se coloca una sonda vesical, obteniéndose habitualmente orinas claras, lo que permite evitar la necesidad de mantener un sistema de irrigación permanente.

La sonda puede retirarse unas horas después, solo en el caso de hacerse la vaporización bajo sedación e infiltración periprostática de anestésico local puede incluso evitarse la colocación de sonda, como preconizan algunos autores. (7).

\section{RESULTADOS}

\section{Seguridad}

En los últimos años han ido apareciendo un número mayor de publicaciones poniendo de manifiesto los resultados de distintos grupos con la utilización del láser verde aunque, en aras de la verdad, hay que reconocer que la mayoría son prospectivos, no randomizados y con un seguimiento relativamente corto, lo que en muchas ocasiones no permite alcanzar conclusiones contundentes.

Aún así, analizándolos en profundidad podemos alcanzar algunas evidencias.

Si investigamos sobre la seguridad del procedimiento, la mayoría de los trabajos ratifican que son muy pocos los efectos secundarios y las complicaciones de la vaporización. 
En cuanto a la hemorragia y por tanto a la necesidad de trasfusión, prácticamente ningún grupo ha precisado trasfundir a ningún paciente, mientras que la literatura asume un $4 \%$ de trasfusiones después de la RTU (8).

Sin embargo hay que aceptar y más en pacientes anticoagulados la posibilidad de hematurias leves, sin repercusión clínica, que pueden mantenerse hasta pasadas varias semanas de la intervención, con un porcentaje que varía entre el 2,5 y el $6 \%$ (9).

Dado que el suero utilizado para irrigación es salino o agua destilada tampoco se describen sídromes de reabsorción, mientras que en la RTU aparecen entre un 0,5 y un $2 \%(10)$.

En consecuencia la retirada de la sonda vesical es muy precoz y como se ha comentado con anterioridad la mayoría de los grupos la retiran en las primreras 12 horas $y$, en algunos casos, si no se utiliza anestesia general o espinal puede incluso obviarse su colocación.

La disuria en el postoperatorio inmediato no está recogida en muchos de los trabajos y los que la reflejan la cifran alrededor del 10-12\% $(11,12)$.

Es también importante considerar, y más en pacientes jóvenes, los efectos secundarios en la esfera sexual, en ese sentido no se han comunicado efectos deletéreos en la potencia sexual de aquellos pacientes previamente potentes

\section{Eficacia}

La mayor parte de los trabajos coinciden en la precocidad con que puede retirarse la sonda vesical, pudiéndose concretar entre las 12 y las 24 horas $(13,14,15)$. En nuestra experiencia a todos los pacientes se les retiró la sonda a las 18 horas de la intervención y solo un $4 \%$ precisó mantenerla 36 horas.

La medición de resultados se ha realizado fundamentalmente midiendo flujo máximo (Qmax), niveles de sintomatología según escalas IPSS o AUA así como cuestionarios de calidad de vida (QoL).

En cuanto a la mejora en el flujo máximo es de constatar que todos los grupos comunican una mejora significativa que llega a alcanzar en algún trabajo flujos de hasta 30,7 ml/sg. (16), aunque en la mayor parte de las publicaciones las medias de mejoras no son tan significativas moviéndose entre 18 y $22 \mathrm{ml} / \mathrm{seg}(17,18)$.

Es también importante destacar que dicha mejoría se alcanza igual con Laser de $60 \mathrm{~W}$ de potencia como con los de 80 W. $(19,20)$.

Si analizamos los datos referidos a la puntuación de síntomas y mejora en la calidad de vida en ambos cuestionarios la disminución de la puntuación alcanza cifras de más de 10 puntos.
Sin embargo en los estudios comparativos con la RTU, que por otro lado son escasos, esta mejora obtenida no tiene diferencia estadísticamente significativa con la que obtiene la reseccción prostática (11), tomando también en consideración que el seguimiento es relativamente corto, habitualmente no más de seis meses (21).

La incidencia de estenosis uretrales publicadas en las series de fotovaporización son similares a las que se publican en relación a la RTU, aproximadamente un $7 \%$, y aparecen fundamentalmente en los primeros 3-6 meses después de la intervención.

Una de las ventajas que se comunican en relación con la vaporización con Laser KTP es la posibilidad de tratar próstatas de gran volumen, en pacientes con comorbilidad, evitando la necesidad de adenomectomías con cirugía convencional, sin embargo la disminución de volumen prostático con esta técnica viene a ser un $40 \%$ de media con un rango entre 15 y $50 \%$. Mientras que la adenomectomía disminuye el volumen en un $80 \%$ y la RTU un $60 \%$.

Estos datos se corroboran tanto mediante ecografía transrectal como mediante la determinación sérica de PSA pre y postcirugía. Es por tanto planteable la posibilidad de que los resultados obtenidos, en términos de mejora de síntomas, pudieran empeorar con un seguimiento más largo, pero como se ha comentado anteriormente no existe un número significativo de publicaciones a largo plazo y, en algunas de la que existen, los pacientes seguidos a más de tres años no suponen más de un $30 \%$ de la series incluidas $(22,23)$.

\section{DISCUSIÓN}

La vaporización prostática mediante Laser KTP se está paulatinamente implantando en muchos hospitales, en España hay instalados 55 equipos (datos de la compañía suministradora) y son cada vez más los artículos que avalan su seguridad y eficacia.

La literatura pone ya de manifiesto que sus indicaciones son similares a las de la RTU, fundamentalmente en cuanto al volumen prostático se refiere.

También se comprueba, en los pocos estudios comparativos con los que contamos, que los resultados que se obtienen en cuanto a la mejora del flujo y la disminución de los síntomas son comparables a la resección transuretral $(24,25)$.

La técnica tiene, por otro lado una curva de aprendizaje corta y por ello está al alcance de la mayoría de urólogos con experiencia en técnicas endoscópicas del aparato urinario inferior (21).

Al mismo tiempo los autores publican que esta mejoría se obtiene con una disminución tanto de la estancia media, el tiempo de sondaje, el porcentaje de trasfusiones así como con la probabilidad de sufrir síndrome reabsortivo $(18,19,20)$. 
Siendo eso así podríamos preguntarnos si la RTU es una técnica en decadencia. La respuesta es NO y ello basado en que la evidencia científica aún no tiene pruebas suficientes en cuanto a la eficacia a largo plazo y, en consecuencia, el posible incremento del índice de reoperaciones a medio y largo plazo.

Hemos de tener en cuenta que la disminución de volumen prostático y el descenso del PSA postoperatorio medido a los 3 y 6 meses de la intervención es mayor con la resección que con la vaporización (11), lo cual puede tener como consecuencia la mayor probabilidad de crecimiento del tejido prostático y el posible empeoramiento de los síntomas con el paso del tiempo.

Así mismo se cuestiona la posibilidad de una correcta técnica en pacientes con próstatas muy voluminosas, que además precisarían de un tiempo quirúrgico claramente más largo e incluso de la utilización de más de una sonda Laser con el encarecimiento de una técnica ya de por sí costosa. El coste del generador está entre los 100.000 y 120.000 € y cada fibra (no reutilizable) entre 1.000 y $1.200 €$. Hoy es difícil, por los entornos tan distintos en los que la sanidad española se mueve, tener datos comparativos del coste de las dos técnicas teniéndonos que referir a publicaciones extranjeras con las limitaciones que ello conlleva (26).

Aunque carecemos de datos publicados, está ya en el mercado un Láser KTP aún más potente de $120 \mathrm{~W}$ que podría disminuir el tiempo quirúrgico y posiblemente facilitar la vaporización de un mayor volumen prostático. Los trabajos con la experiencias preliminares estarán a nuestro alcance a lo largo de este año.

Habría que preguntarse también si, puesto que es un tratamiento seguro, eficaz y con una tasa muy pequeña de complicaciones, habría que contemplarlo como alternativa al tratamiento médico y más en aquellos varones en los que pudiera estar indicado un tratamiento combinado con alfa-bloqueantes + inhibidores de la 5-alfa reductasa, dado el coste a largo plazo que ello conlleva y que, aunque poco frecuentes y poco importantes, esta terapia también presenta efectos secundarios. Como en los comentarios anteriores necesitamos de estudios comparativos que puedan contestar dicha pregunta

\section{CONCLUSIONES}

La fotovaporización prostática con Laser KTP es un procedimiento seguro, con una curva de aprendizaje corta, con escasa morbilidad e igual de efectiva, a corto plazo, que la RTU para el tratamiento de la HBP con síntomas obstructivos.

Es preciso que trascurra más tiempo para confirmar que dicha eficacia se mantiene con el paso de los años.

El desarrollo tecnológico empieza a proporcionar energías Láser aún más potentes que parecen disminuir el tiempo quirúrgico y abordar próstatas de mayor tamaño.

\section{BIBLIOGRAFIA y LECTURAS RECOMENDADAS (*lectura de interés $y^{* *}$ lectura fundamental)}

1. COSTEllo, A.J.; BOWSHER,W.G.; BOLTON, D.M. y cols.: "Laser ablation of the prostate in patients with benign prostatic hypertrophy". Br. J. Urol., 69: 603, 1992.

2. KUNTZ, R.M.: "Current role of laser in the treatment of benign prostatic hyperplasia”. Eur. Urol., 49: 961, 2006.

3. BHATTA, K.M.: "Laser in urology". Laser Surg. Med., 16: 312, 1995.

4. KUNTZMANN, R.S.; MALEK, R.S.; BARRET, D.M. y cols.: "Potassium-titanyl-phosphate laser vaporization of the prostate: A comparative functional and pathologic study in canines". Urology, 48: 575, 1996.

5. KUNTZMANN, R.S.; MALEK, R.S.; BARRET, D.M. y cols.: "High-power $(60 \mathrm{~W})$ potassium-titanylphosphate laser vaporization prostatectomy in living canines and in human and canine cadavers". Urology, 49: 703, 1997.

*6. MALEK, R.S.; BARRETT, D.M.; KUNTZMANN, R.S.: "High power potassium-titanyl-phosphate (KTP/532) laser vaporization prostatectomy: 24 hours later". Urology, 51: 254, 1998.

7. CHANDRASEKERA, S; MUIR, G.: "Potassium-titanyl-phosphate laser prostatectomy: A review". Curr. Opin. Urol., 17: 22, 2007.

8. AHMED, H.U.; THWAINI, A.; SHERGILL, I.S. y cols.: "Greenlight prostatectomy: A challenge to the gold standard?". Surg. Laparosc. Endosc. Percutan. Tech., 17: 156, 2007.

9. FU, W.J.; HONG, B.F.; YANG, Y. y cols.: "Photoselective vaporization of the prostate in the treatment of benign prostatic hyperplasia". Chin. Med. J. Engl., 118: 1610, 2005.

10. OKEKE, A.A.; LODGE, R.; HINCLIFFE, A. y cols.: "Ethanol-glycine irrigating fluid bsortion in transurethral resection of the prostate in practice". BJU Int., 86: 43, 2000.

*11. BACHMANN, A.; ACHÜRCH, L.; RUSZAT, R. y cols.: "Photoselective vaporizatiopn (PVP) versus transurethral resection of the prostate ( TURP): A prospective bi-centre study of perioperative morbidity and early functional outcome". Eur. Urol., 48: 965, 2005.

12. KUMAR, S.M.: "Photoselective vaporization of the prostate: A volume reduction analysis in patients with lower urinary tract symptoms secondary to benign prostatic hyperplasia and carcinoma of the prostate". 173: 511, 2005.

13. VOLKAN, T.; IHSAN, T.A.; YILMAZ, O. y cols.: "Short term outcomes of high power $(80 \mathrm{~W})$ potassium-titanyl-phosphate laser vaporization of the prostate". Eur. Urol., 48: 608, 2005.

**14. MALEK, R.S.; KUNTZMAN, R.S.; BARRETT, D.M.: "Photoselective potassium-titanyl-phosphate laser vaporization of the benign obstructive prostate: Observations on long-term outcomes". J. Urol., 17: 93, 2005.

15. BARBER, N.J.; MUIR, G.H.: "High-power KTP la- 
ser prostatectomy: The new challenge to transurethral resection of the prostate". Curr. Opin. Urol., 14: 27, 2004.

16. HAI, M.A.; MALEK, R.S.: "Photoselective vaporization of the prostate: Initial experience with a new $80 \mathrm{~W}$ KTP laser for the treatment of benign prostatic hyperplasia". J. Endourol., 17: 93, 2003.

17. SULSER, T.; REICH, O.; WYLER, S.: "Photoselective KTP laser vaporization of the prostate: First experiences with 65 procedures". J. Endourol., 18: 976, 2004.

18. SANDHU, J.S.; NG, C.; VANDERBRINK, B.A. y cols.: "High-power potassium-titanyl-phosphate photoselective laser vaporization of prostate for treatment of benign prostatic hyperplasia in men with large prostates". Urology, 64: 1155, 2004.

19. MALEK, R.S.; KUNTZMANN, R.S.; BARRETT, D.M.: "High power potassium-titanyl-phosphate laser vaporization prostatectomy". J. Urol., 163: 1730, 2000.

**20. ALEXIS, E.; MALLOY, T.R.; STEIN, B.S. y cols. "Photoselective vaporization of the prostate for the treatment of benign prostatic hyperplasia: 12-month results from the first United States multicenter prospective trial”. J. Urol., 172: 1404, 2004.
21. HEINRICH, E.; SCHIEFELBEIN, F.: "Technique and short-term outcome of green laser (KTP,80 W) vaporization of the prostate". Eur. Urol., 52: 1632, 2007.

22. GILLING, P.J.: "Laser vaporization of the prostate: Are we there yet?". Eur. Urol., 52: 1569, 2007.

23. TE, A.E.; MALLOY, T.R.; STEIN, B.S. y cols.: "Impact of prostate-specific antigen level and prostate volume as predictors of efficacy in photo-selective vaporization prostatectomy: Analysis and results of an ongoing prospective multicenter study at 3 years". BJU Int., 97: 1229, 2006.

**24. HORMINGER, R.M.; UNTERLECHNER, H.; STRASSER, H. y cols.: "Transurethral prostatectomy: Mortality and morbidity". Prostate, 28: 195, 1996.

**25. HOFFMAN, R.M.; MacDONALD, R.; SLATON, J.W. y cols.: "Laser prostatectomy versus transurethral resection for treating benign prostatic obstruction: A systematic review”. J. Uro., 169: 210, 2003.

26. STOVSKY, M.D.; LASKIN, C.R.; GRIFFITHS, R.I.: "A clinical outcome and cost analysis comparing Photoselective Vaporization of the Prostate (PVP) to alternative minimally invasive therapies and TURP for the treatment of benign prostatic hyperplasia”. J. Urol., 171: 393, 2004. 